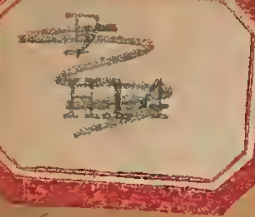

A FEW

Scripture Selections

and Hymns.

In the Hidatsa or Gros Ventre Language

\title{
2nd Edition.
}

Published for the $P M / 331$
$Z 71 / 2$
1906 FORT BERTHOLD MISSION

EImowoods No. DAK.

$$
1 \overline{906} \text {. }
$$









\section{A FEW}

Scripture Selections

\section{and Hymns.}

In the Hidatsa or

Gros Ventre Language

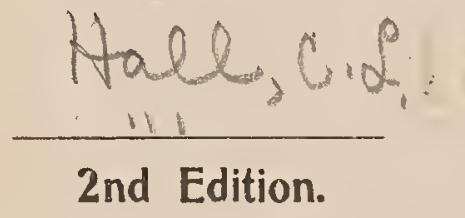

Published for the

FORT BERTHOLD MISSION

Elsowoods, No. DaK.

1906 . 


\section{ppr}

This book contains a few passages of scripture and a few hymns for the use of Christians understanding the Gros Ventre language spoken at the Fort Berthold Indian Agency-Elbowoods, N. D.

It is hoped that they will learn to read, and commit to memory the contents of these pages, and thus carry about in their hearts some of the thoughts of God.

Mr. C. W. Hoffman has written a number of the hymns; G. K. Bassett wrote two.

In all the Bible translations, Edward Goodbird has been an efficient colaborer.

C. L. Hall, Editor. 


\section{The Gros Ventre Alphabet.}

VOWELS.

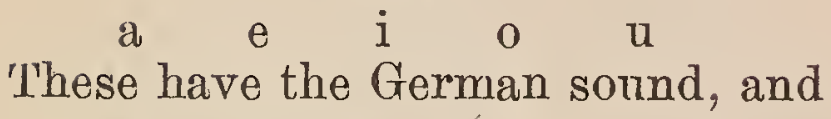
are long and short.

\section{CONSONANTS.}

e. $\mathrm{h} k \mathrm{~m}$ w $\mathrm{p}$ s $\mathrm{t}$ pronounced as in English.

$h$ is a gutteral sound.

ś equals sh in English.

, is an aspirate or breathing at the end of a syllable. 


\section{John 3: 16.}

Mahupai'tiaś ik awa hakaheta kideśits, heeśawa idiśa kaduśewa akuikapati'ka kaheś, heeśawa tapediaduśa šeetahaaduk adukideśe'ketaats, hi maaduiidi aduataka desa aduaats.

\section{John I: 7.}

Idiśa Jesus ihua aduidi'ta maadutsawi aduiśia adumahoo etsa imikiduśukaats.

\section{Jesus Itamaadukiweke-The Lord's Prayer.}

Matuo akua'pahikua, Didaśi aduhupa'kaats. Ditadutsawi huhits. Ditaduiśke awa hedu hidihats, a'pahikua iwakśeśatsa. Mapehe maadumutoo mikuada. Heśak maaduiśia adumahoo kiduśka mikuada; akumiiśihoo makiduśuka makuidu he iwakśeśatsa. Aditaśe miakdahitada; haśa maaduiśiakua awa'kuaduk mikutsada.

Maadutsawi he di ditawaeets, heśak maaduakaki he, heśak maadukiwade, ataka deśa. 


\section{Mahupai'tia Itadutsawi Pidaka.}

\section{The Ten Commandments.}

1. Maśokidu mahupa iha deetadits.

2. A'pahi ditaakadu maaduiwakśeśatsi dahetadits, militadu awakua, hak midi awa awahukaduś.

Śio iśokidu mikadatadits heśak diitawaśidiata, Jehowa ditamahupa mits. Mi mahupa akumaakawiahtekśats. Atuo maaduiśia aduhidoo itamakadiśtakua odiwats, adui'kidakapatoo idami itopakua akumiiku'pao śiata. Heśak mikideśak mataduiśke hidaaduk, maaduikapati pidaki'tia akakodi mamadatiśiamits.

3 Jehowa ditamaliupa he daśi išatsa imaiakadatadits. Jehowa daśi iśatsa akuimaiakahoo adutsakihetats.

4 Mapi iśapua he aduhupa'kidats kadap. Mapi akama madahedits, heśak maodahe etsa dihadidits, haśa mapi iśapua he Jehowa dita mahupa he itawaeets. Adumadahetats śedu, dik, didiśak, di'kamiak, ditaśikakak, ditamiakaśak, ditamitek, iśa datikutataduś. Jehowa a'paliik, awak, midi'tiak, 
hak śeekua mamaki etsa hidits. Heśak mapi iśapua hedu otsi'kets, heeśawa Jehowa mapi iśapua be tsaki' kets heśak hupa'kets.

5 Datuk, dihuk, ditamadei'tidadits; haduk Jehowa ditamahupa hedi awa akudikudu tia daha'kudits.

6 Matidatadits.

7 Iha dakhapitadits.

8 Maaśadita.

9 Ditadohpakata maadumitapa imadidetadits.

10 Ditadohpaka ati adaatadits. Ditadohpaka ua adaatadits. Itasikakak, itamiakaśak, itamitek, itaśukak, itatapaduś etsa adaatadits. 
Maadutsakihe.-The Beatitudes

Matt. 5:3-12.

Tawii idahikua kidaśatsiduk śio adutsakihaats. A'pahikua maadutsawi itawaaats.

Tawii iwiiaduk adutsakihaats, śio adukidaśtaats.

Tawii adutsawi hahehiśatsiduk adutsakihaats, śio awahe aduitawaaats.

Tawii maadutsawutsi iaditak iiutsaaduk adutsakihaats, śio adumailipataats.

Tawii madatiśiaduk adutsakihaats, śio odatisiaats.

Tawii madatakuhaduk adutsakihaats, śio Mahupai'tiaś aduikaats.

Tawii akumamakiitadohpake'kaaduk adutsakihaats, śio Mahupai'tiaś itamakadiśta aduiidaśataats.

Tawii dohpakidi dimakak, dimahidapidi'kaaduk, heśak mitapak maaduiśia etsa diśiaduk miiutak, oditsakihaats. Diapak didatatsakikatada. A'pahikua ditamadu aki'tiats. Diheśetakua matse hupaaś dolipakidi iśa mahidapidi'kaaśta. 


\section{Maitu. Psalm 23.}

1. Jehowa mataaśi'tia akuika śeets : maduwa adumamahetats.

2. Mika adutohikua mihapi'kets; midi aduhaheiśata itukak miakdeits.

3. Madahi kitsaki'kets. Maadutsawutsi adiś śeduha miakdets, daśiś śeeta.

4. E! Maadute aśiś aduhapeiśaśe madididuś, maaduiśia, adumakiatats, midikupawa. Ditamidakaśak ditsakik mikitsoki'kaats.

5. Maśokidu maoduti daha midakuts, matamaihaaś itadu. Tsadata matu tsatidats; matamidiihi'ke mahua ha'kuts.

6. Kati maadutsakik maodatiśiak mapi tuaka maha'kudak, miapi odadiats, heśak Jehowa atidu aduwawakuts ataka deśa 


\section{Maitu. Psalm 32.}

Tape maaduišia aduhidi kipakiśiduk, heśak maaduikahti itipeduk, seedi adudatatsakits.

Dohpakaś odatatsakits, tape Jehowa maaduikahiti adukiduwitats, heśak data adumitapa deśats.

Madeta wawa'kuwa miaduhidu utsa dets ; mapi hakaheta mamidakak. Mapik oktsiak diśakidi awata mipakidiats.

Mahua idi awaade adukiutsi iwakśeśatsa dets. Matadutsawi aduišia dimakiwets; heśak maadumikahti makaliuatats: Maadumikahiti śio Jehowa adumakiwets, wats. Heśak maaduiśia maheś dakiduśuka midakuts.

Heeśawa tawii matapadatsaaduk etsa odikiwekaats, tuaka diodapáaduk. Kati midi uahe i'tiaduk, śiota aduuśiataats.

Mataduiahu dits. Maaduhidapi watuduk midakutsidits. Maaduidi'ke maitu mihopidadits. 
Maowia madikumits, heśak adi tota odade dimakiwewits. Dimakupa mademits, maśtata diamakamits.

Itsuwaśukak, api'tiak, śio mae'ketats, odiśeśatsitats. Hak uwetsa iidutik. aduiliuiśik śiota aduidadukidiats, śeeśa deśaduk odiutidu odahutaats.

Tawii isiaduk maaduhidapi ahu aduaats; haśa tapeduś Jehowa śetaheduk, maodatiśiadi aduhupa a'kuats.

Tawii tsawutsa ada'kuaduk, Jehowa śeekua odiepak, odidatatsakaats. Heak tawii datatsawutsa ada'kua etsa diepak oditsatskaats. 


\section{Christ aduiwatu.}

\section{The Birth of Christ. Luke 2:\$- 20.}

Awa kaduśedu aśii'tia akuikaś ita. mahakawa'ku aduikakua aka'kuats. Heśak Mahupai'tiaś itawaśi itaankadu hits, heśak Mahupai'tiaś itamaaduateiśa itaiahia ikaditskats; heeś:?wa kia ihidaats. Heeśawa Maloupai'tiaś itawaśi śeedi maśets: Kietada; heeśawa ika, maadukiwe adutsaki, maaduiepi aki'tia makmahu madikuats, dohpaka aduśukets. Heeśawa mapehe akumaidi'keś ikapata dikuats, David awatikua, śee Christ śeets, akumatseitsiś. Heeśawa maaduilyata dido'ta hidits: makadiśta adukidakśi wa aduadodapaats, itsuwaśuka ee iśikua wakits. Hesak Mahupai'tiaś itawaśi duwetsakua ikupapak ikiaki'tiats Mahupai'tiaś śeeta kiwedaats; śiats: Mahupai'tiaś itamaaduateiśa akumaku kati; heśak awakua tawii Mahupai'tiaś itadutsawi kidetaduk, śio adukiduwetsihaats. 


\section{Christ adukitahaaš.}

\section{The Crucifixion. Matt. 27;33-50.}

Golgotha akuhaats śedu uśiawa(awa maatu hiśeś śeduts) midiadawi kuats, adapadi iaśahak heśak datiśak ihitats. Heśak midamakipihedu daktadia śedu ihuiśi makiimakitsaats imakimakiiats. Heśak śedu amakak kikeśa kats. Hesak atu akata patsak itaankaduha maaduakahoo akakaśaats: Hidi Jesus śeets, Jews itamatseitsaats. Heśak dohpaka akumaaśadoo dopa midamakipihedu daktadiats, Jesus ikupak, duwetsa itaidapakuha dakits, heśak duwetsa itaidakiśakua hets. Heśak tawidi odaaduk akuataats, heśak atu ihakahahaats, heśak śiats; Di'ki makiweke-atiś dakidutsitak mapi damiduk iśa dakikśi damahets, dikiidi' kedi; Mahupai'tiaś diidiśaduk, midamakipihe e'kuaduhak kidapak hu, haats. Mapatsi okuahaaś siadik, hi akumaakakaśaaś ikupak, heśak akumadei'tiaś siadik, idoki iśa heśatsa akuatak śiats: Aduihaś adukiidi'kets, iiduk adukiidi'ke aduakakitats 
Israel itamatseitsiduk midamakipihe e'kukahak kidapak huduk, makadatsemihats. Mahupai'tiaś miidišats, Jesus heśta, heeśawa śiats. Heśak akumaaśadaaś iśa api midamakipihedu akudaktadiaś itamaaduakahoo iwakśeśatshaats. Miảiikikiśkia aduakamaduhak awa hapehiśats heśada oduwetsapi kuats, heśak oduwetsapi śiaka aduhiwa Jesus hi'taha pak śets: Eli Eli lama sabacthani, iśa śets, Matamahupai'tia, Matamahupai'tia tośewa mihadata? Heśak dohpaka duwa śedu kaats ikikuak śiats: Hidi matse Elijah daśatits; hesak ik kaduśeśa duwetsa tidiats, heśak midi-iduhipe dutsak midihikuakua opśakak, pu'puakua dihak heśak hi'kets. Heśak aduhidi'tadi siats: Hakata Elijah huak kiidi'keduk amakamihats. Heśak Jesus iśa i'taha pats, heśak idahi de'kets. 


\section{Maadukidahi.}

\section{The Resurrection.-Matt. 28.}

Atade mapiliupa aduheda itamapi itsi 'ka, Mar'y Magdalene hi Mary aduiha śiak, dokte-oduśa ika uśiats. Heśak ika śedu awadada i' tiats; Mahupai'tiaś itawaśi a'pahikuhak awata. hits. Heśak uśiak miiś patota dutsita dihets, heśak ankadu amakits. Aduika kadr'ka hiśets, heśak ihuiśi ohata i ma hiśets. Heśak Mahupai'tiaś itawaśi śedi aikuphits, heśak miaś śeta maśets: Kietada, Jesus dakikidawa ema'kets, midamakipihedu akudaktadiaś śee wakits. Hidoo wa'kitats, see kidahits aduśe iś heśets. Dahuada, akumatseitsiś aduwa'kiś śedu ikada. Heśak diśidiśa kata dahadak, itaakumakikutskaaś makiwa: Kidahits adutekuhak, heśak ika Galilee seta diśokadu dets. Śkekna aduadakaats Ika dimakiwaaśta. Heśak hatataka dokte - oduśa śekuhak dutsita dahak, kiaśa iepak i'tiats, hak tidiak itaakumakikutskaaś śiata dokade akdahuats. Heśak ika Jesus mauśiak sets: Diepada. 
Heśak śeeta uśiak itsi akśiats, heśak seeta kiwekaats. Śiaka Jesus śeedi maśets : Diiditeta, matametsaś da makiwa, śeduha Galilee 'śeta adudahats, śekua adumiikaats Matt. 28:1-10.

Akumakikutskaaś iahpiduwetsa Galilee śeta dahats ; awa akumakuś Jesus śeedi akuowiaś śedu adumai'kopi maiheś ; heśak Jesus ikaawa śedu iśookidu mikahaats; haśa duwadi okśatsedetsaats. Siaka Jesus mahiak iśa śets: A'pahikuak awaheduk maaduitsia etsa mikuats. Heeśawa dahada, dohpaka etsa matadut,sawi makikutski' kada; Atuk, Idišak, Akuidahihupa daśi śiata imaati midiakipabuada. Owiakahada madiki'ke akumadikuaś adukudaats; heśak kadawada, mik odimakupats tuakaduśa adumapi ihakakua aduihits. Matt. 28:16-20. 


\section{Idišaś Kits.}

\section{The Prodigal Son. Luke 15: 11.}

Heśak (Jesus) śets: Matse wa idiśa dupats; heśak okadiśtidi atuś śeeta iśa śets: Tate, maadumiitawaee'kida ditamadowae mi'kuk. Heśak itawao i'kimakitsets. Heśak itiata idiśa okadiśta itawae etsa kiduwetsi' izets, heśak awa adutiaśita dadak dets. Heśak śekua itamadowaaś madu'tak etsa kawahetets. Heśak etsa kawahetak, hak awa śedu maaditi i'tia katets, heśak kiadiaśatsi hidits. Heśak awa śedu akua'kuata dak aki wa'kuts; heeśawa adukatikua waśiitadahpitsi maduti'kekaats. Heśak waśi itadahpitsi itamaodutaś ik ii'pati itets; heśak tapedidus maduwa kutats. Haś i'kadawatsak śets : Tatiś itawaśi aki'tia iś ; ehaduśa maoduti ahu iś ; haśa hidoo madita mata dets. Imiduhimits heśak tatiś śeekita mademits, heśak iśa sewawits: Tate, a'pahitak, di'ta maaduišia mahets; heśak hidikak madiśa adumidatats; ditawaśo e'kua adumiiwakśeśatsidats, wawits. Heśak ii- 
duhak atuś śeekita kuts. Haśa hidakatsa tiaśa duwiha atuś śeedi ikats, datiśiats; heśak tidiak śeeta dets hak apadahpak i kidatsopits. Heśak idiśaś śeedi śets: Tate, a'pahitak, di'tak maaduiśia mahets, hesak hidikak madiśa adumidata ha'kats. Haś atuś śeedi itawaśaaś śiata śets: Maśi akutsaki diśidiśa kata akdahuada, heśak i'kada, heśak śaki ioptsati dihada, heśak itapa u'kada. Heśak wite idaka akuidipi akahuk kitahada. Heśak mutimihats, heśak matsakihiśe'kiwawihats. Hidi madiśa he teśta haśa kiidits; hapats heśak hidi okipapaats, hets. Heśak matsakihiśe' kaats śiaka.

\section{John 20: 30-31.}

Jesus maaduiiyata ahu hidits itaakumakikutskaats śiata, hidoo maakakaśi hedu akitats ; haśa hidoo akuakakaśa kahe, Jesus Christ śeets Mahupai'tia Idiśaś kukaha aduidakadatsaats; hak dakadatsaaduk daśi hekuha odiidaats. 


\section{Jesus Ita Maoduti Hipa}

\section{The Lord Supper. Matt. 26: 26-30.}

Madutawa śedu Jesus madahipe dutsak maaduiepi śiak, heśak duliuhits ; heśak itaakumakikutskaaś śiata kahets, heśak sets: Dutsak, dutada, hidi mahuats. Heśak midihi'ke dutsak, heśak maaduiepi śets, heśak kahak sets: Hiada etsidi; hidi maliua idits, maadukiduwetsa'ke aduhidats, hidi akupaliua he dohpaka iahuaduśa itamaadutsawi aduiśia adukiduśnkits. Haśa didota śewats: Hidika maśipiśa adumidi oduwatats. Tuaka tatiś itaoda kikua muuśiaduk śiaka dimakupapak aduhida aduwats. Heśak maitu pahak atadak dahats, Olives awa maku seeta.

\section{Maadutsakihaaš.}

\section{Benediction. 2 Cor.13-13.}

Jesus Christ matamatseitsi itamaodatiśiak, Maliupai'tiaś itamaadukideśik, heśak Akuidahihupaś itaadumakiidakua, tuakaduśa etsa odiikupaats. Amen. 


\section{Maadukiwedaaś Doxology.}

Matuo a'pahikua

Kiwedada; Idišaś Christ,

Hak Akuidahi-hupaś,

Di etsidi katetsada.

Tośe Hiša wawa'ku.

Just as I Am.

1 Mik tośe hiśa wawa'ka,

Matadutsawiśia ahu

Diśokidu madahadets,

Jesus di'ta adumahuts.

2 Mik tośe wawa'ku mahuts,

Matadutsawiśia ahu

Dakiduśuka midakuts,

Jesus di'ta adumahuts.

3 Mik tośe wawa'ku mahuts,

The midakikiwiduk,

Matadutsawiśia ahu

Adumaduśats hidika.

4 Mik tośe wawa'ku mahuts,

Ditadutsawi śe tsakits,

Mik iniwawits hidika,

Jesus di'ta adumahuits. 


\section{Awa Itamaaduamahati.}

\section{The Light of the World.}

1. Awa hakaheta hapehiśaśta,

Śe amahate maihets.

Mapi midi adukaditski hiśats,

Se amahate maihets.

Dahuada, Jesus śetahak

Wate Jesus miamahatets,

Dahuada, Jesus śetahak

Di amahate maihets.

2. Adutsawi iśia hapeiśi'kaats,

Se amahate maihets, Jesus śe etsa adukitsaki'kets Śe amaliate maihets.

3. Jesus ikupaduk hapeiśitats, Śe amahate maihets Adu amahati śe miakdahaats, Miamahate maihets.

4. A'pahikua mapi midi da' kitats, Se amahate maihets, A'pahii itamahati Jeśus śets, Se amahate maihets. 


\section{Dita Midamakipe.}

\section{I am Coming to the Cross.}

1. Dita mida makipe, seta hidika mahuts, Mamadata hita its, Etsa daha midakuts.

Matseitsi di'tawats, Miiutak datiśta, Jesus mididatiśiats, Miidi'ka hidika.

2. Dit adi adutsaki

Mik madahiiśets haśa

Adowia midaku,

Ema'kemits hidika.

3. Kidiadopatikua

Maliua diwats hidika;

Maiśia mahets haśa,

Kiduśuka midakuts.

4. Mata hidapits haśa

Mik madets ditadikua.

Mitsakidi madetats,

Dik midakikiwidits. 


\section{Maaduidi Adi.}

Rock of Ages.

1. Maaduidi adi,

Jesus di'ki dits adi,

Heeśawa di'tawats

Adu di'ta makadats,

Di'ki ditahaawa

Mi idits, śeekuha.

2. I)ita maadikike

Mie'kehada tate

Odidehe omiya muk,

Odidiyahi hu'ka muk,

Heeśaduk śeedi

Miidi'ke akakits.

3. Di adutsawi tsakits,

Hehawa dita maits

Mapi etsa mamahak,

Mapi etsa mimiyaduś

Adumitsaki'ketats,

Josus dik adakakits.

1890,

G. K. B. 


\section{Jesus Itsakita Itsiats.}

\section{My Hope is Built on Nothing Less.}

1. Dohpaka adutsawihe Awahedu maakakits;

Aku'ta akakitaits Maaduśeetahetaits.

Jesus itsakita itsiats ; Hidita etșa deśaats, Hidita etsa deśaats.

2. Mahiupai'tia he i'ki Maakakita deśaits. Maaduśe heeśaits : Kiilia'ke maihetaits.

3. Tawii Jesus kadatseduk, Heśak kiwakaaduk, Sio tsatsuka kahaats Miihapa he akadu.

4. Etsa mahupa ihata Tata adukideśaats ; Midi detakua puhaka Iwakśeeśatsihaats. 


\section{Jesus Mikikuhak. \\ I Hear Thy Welcome Voice.}

1. Jesus mikikuhak, Di'ta adumahuts; Dit idita mikiduśuk, Dit idi pahuaś.

$$
\begin{aligned}
& \text { Jesus di'tawak, } \\
& \text { Di muuśiats. } \\
& \text { Di idita miduśuk, } \\
& \text { Dit idi pahuaś. }
\end{aligned}
$$

2. E, mitsatsukitats,

Dik mitsatsuki'ka.

Mi śipiśa miohata,

Ma iwakśeśatsa.

3. Jesus mikikuhak,

Di mikikuamits ;

Idimitia wawakuts,

Aku mieeś dits.

4. Ikaka idi he!

Ika midatiśiats.

Ika matseitsi i’tia.

Matawaees dits. 
5. Jesus mikiduśuk, Dit aduśe tsakits. Mamiadadaśatiśmuk, Awakulta madoots.

$$
\text { C. L. H \& G. K. B. }
$$

\section{Maadukiwe Mikikua. I think when I read}

1. Maadukiwe mikikua makats, A wakua Jesus da'kuśta, Makadiśta watekua apadahpats, Mik aki miaki awaats.

2. Śee śakita maśeda patipiduk, Ik mapadahpaduk heśak Aduśe mikikuaduk ku awaats, Makadiśta dahuada hets.

3. Wate i'ki a'pahikua da'kuts Haśa śeta makiwekets. Mikideśiwa itaduiśke mahets. Ik itu'ta mik amakamits. 


\section{Jesus Itsaki Kadatsa.}

\section{Only Trust Him.}

1. Jesus iihua katita

Mapatsa dikuśta,

Kaduditsakitaduśa

Jesus dimaihets.

Jesus śetsaki kadatsa

Śetsaki kati;

Diidi'ke, diidi'ke,

Diidi'keśta.

2. Jesus adi tsaki'keśta,

I adutsawi'ta.

Maadudatatsaki'ta

Wate diowiats.

3. Jesus okati śeeśta;

Dik tata kadatsa.

Jesus diakuhti katits,

Jesus dimaihets.

4. Jesus śets: Amahati mits.

Mi'ta dadahuduk,

Odidahitaaduśa

Kawahatiwawits.

C. W. Hoffman. 
Jesus Midakidesits.

Jesus Lover of my Soul.

1 Mata-adutsawita,

Aduimahakuta,

Mahua hetaduśa

Awa iwakśeeśats.

Jesus mikuhti kati;

Dita-adutsawita,

Maaduiidita

Śeeta miekeka.

2 Jesus midakideśits,

Di'ta aduwawakuts.

Ditsaki dihupaśta,

Ku'ta dimakadatsats.

Jesus maaduteta

Etsa itada'keśta ;

Ku'ta maaduteta

Aduśe makiatats.

3 Jesus ditsaki kati

Mieda'ke katıśta.

Maaduiśiata

Mi kati wawa'ku śa,

Jesus duwa miika.

Di'ta makadatsemits.

Tuaka wawakuduk

Di kati miidi'ka.

C. W. Hoffman. 


\section{Diutidu Jesus.}

Nearer My God to Thee.

1. Di utidu Jesus.

Di utidu

Midamakipedu

Miduha aśa,

Mamapaha makats,

Di uta wawaku,

Di utidu.

2. Awaśa madeduk,

Oktsiaduk,

Miiśe mahapits,

Mitsaki śa,

Mamaśiadeduk,

Di uta wawa' ku,

Di utidu.

3. Śsedu adi atets

A'pahita,

Mididatiśiats

Tuakaduś.

Ditidahi hupa

Mikikuhats dita.

Di uta wawa'ku,

Di utidu. 
4. Atawa mitshewa

Di awaśkets.

Maaduhidapi

Watuts aśa

Di adupatikua,

Di uta wawa' ku,

Di utidu.

5. Maaduiepi

Iniwaduk,

Ditsaki di'tawak ;

Tuakaduś

Mamapaha makats,

Di uta wawa'ku,

Di utidu.

1902.

C. L. H.

\section{Daha kuada Jesus. \\ Stand up for Jesus.}

1. Daha kuada Jesus.

Midamakipe he

It akumakikua

Didoki ada'kuats.

Ita madakapilie

Makuka duhada;

Ita maiha etsa

Aduitadakets. 
2. Daha kuada Jesus. Maadumakia

I'tiata dahada, Tawii di matsiaduk.

Maadukiki'tadsi

Watuduś didatoo

Kitsoki'ka kuada, Jesus śetahada.

3. Daha kuada Jesus. Diikupapak dets, Odidotsakitaats, Diiditetada.

It aduimakia

Aduhupa watuts. Etsidi inihada. Jesus i'ti'kada.

4. Daha kuada Jesus, Śeta kiwekada. Idahi Akuhupaś Etsa adukahets. Awa he hakaheta Aduitawaets :

Hak akumatseitsi Okati Christ śeets. 


\section{Didasi Hilupakiwaats.}

\section{Hallowed Be Thy Name-Dulcimer II: 8.}

1. Jehowa didaśi hupakiwaats,

Hak awaheduśa dahets. Apalita etsa di kati dahets;

Dohpaka etsa daheśta.

2. Madahi mahua heduśa dahets.

Sio tsakiha kudaa.

Mi kati maaduliaśa wawa'kuts,

Heśak maemaketaśta.

3. Jesus didaśi adumiekedats

Dita-adưideeta,

Dita-adutsawi apahikua

Adowia midakuśta.

4. Maadutsawi aduiśia he A wahedu ahu katits; Heśak maadudataiśia he Jesus śkuha mikulita.

5. Jesus di'ta maaduiśia deśats ;

Heśak maadute deśats ; Ku'ta di'ki miidike maiha;

Didaśi he makadatsets. I904.

C. W. Hoffman. 


\section{Jesus Madata Tśaki'ka. Jesus Renew my Heart.}

Dakota Hymn No. 6-Tappan.

1. Jesus tsakiha mikuda. Dita-adikua Adowia midakudits. Dita-aduideeta

Ema'ke mamahets.

2. Jesus ditsaki dihupats. Maaduiidi'ta, Maidahi-hupa śeeta, Maadutsawi'tak etsa Jesus dik dakudets.

3. Jesus madata tsaki'ka. Mata-adutsawi Tsawutsa adudakiksits. Maaduitsia katita Śt imididi'ka.

4. O, Jesus midakideśits. Di dibua midakuts. Madahi ikitsaki'ka. Maaduiśia mahe Iiha'taha muk. 


\section{Jesus mikudaa. \\ Hide Me 0 my Savior.}

1. Jesus di'ta mikudaa

Aduliupata.

Ku'ta aduotsi'kiwats

Adutsakikua.

Mikudaa,

O Jesus mikudaa

Aduliupata,

Heśak mitsaki'ka.

2. Maaduhidapikua Muuśiaduk,

Di'kita adumakadats,

Maadukuhtita.

3. Madataiśiaduśa

- Di'ta mahumits ;

Heśak madataiśketa

Jesus tsaki'ka muk.

4. Jesus di'ta mikudaa, Maema'ketats ;

Ditaadutsawikua

Jesus mie'keka.

1902.

C. W. Hoffman. 


Microfilm:

Proesveration

Services

$$
\min _{7 \text { Novas }} \frac{6536.07}{\text { Nov }}
$$


Whiten-1

Prementi

Services

mim 4.536 .07

TNovas 
SANTEE NORMAL TRAINIHG SGHOOL PAESS,

(INOIAN)

SANTEE, NEB\&ASKA. 